\title{
Session I: Biosafety Research and Risk Assessment
}

\author{
Sally L. McCAMMON*
}

Biotechnology Regulatory Services, Animal and Plant Health Inspection Services, United States Department of Agriculture, Riverdale, Maryland, 20737, USA

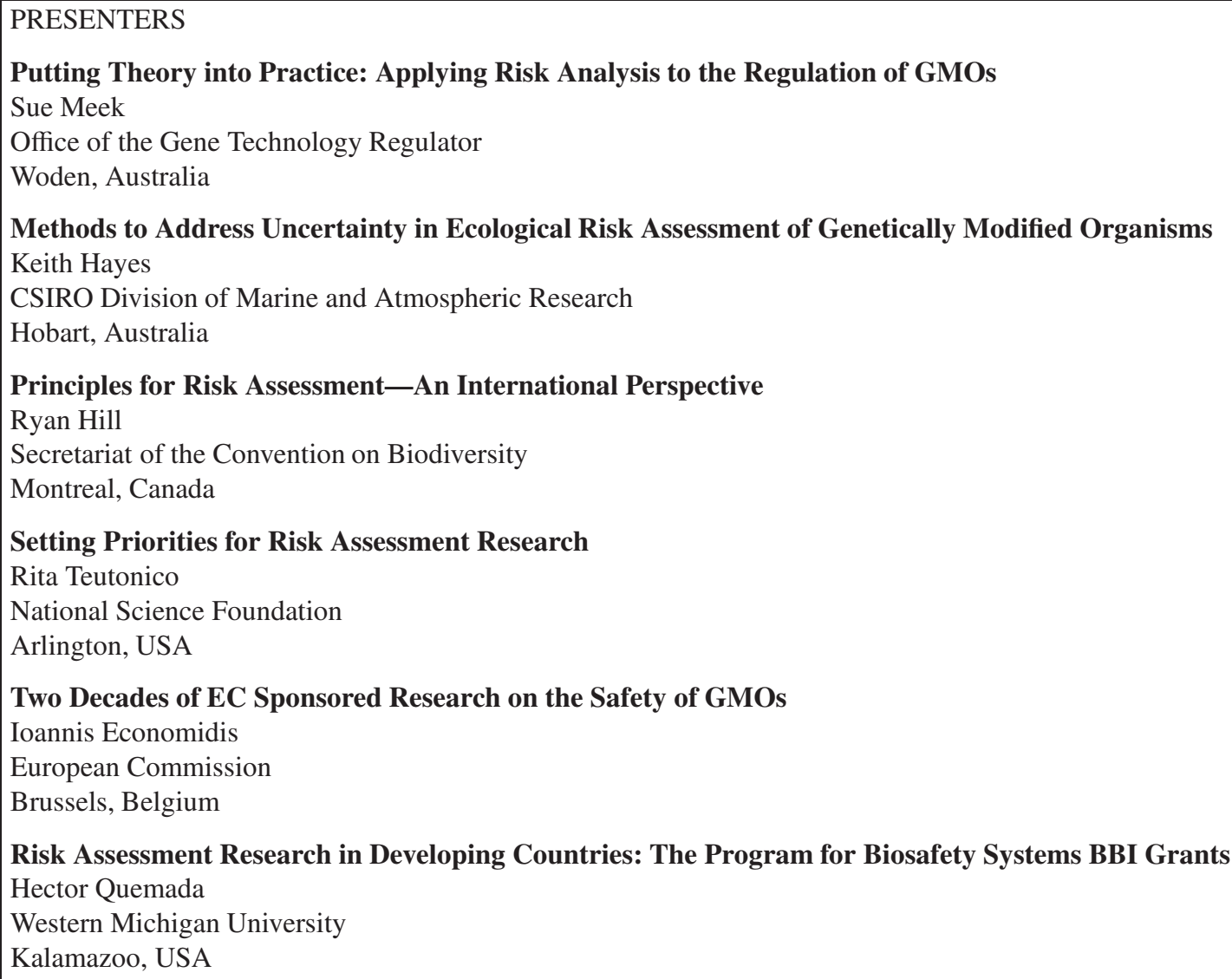

Risk Assessment Research in Developing Countries: The Program for Biosafety Systems BBI Grants Hector Quemada

\section{INTRODUCTION}

International and national guidance on environmental risk assessment provide critical direction about the types of scientific data needed to evaluate transgenic organisms. This guidance by governments on risk assessment evolves over time and is strongly influenced by the results of biosafety research. In turn, national and regional research priority areas are identified based upon close collaboration with risk assessors as their needs evolve. The

* Corresponding author: Sally.L.McCammon@ aphis.usda.gov 9th International Symposium on the Biosafety of Genetically Modified Organisms (ISBGMO) explored the link between biosafety research and its use by risk assessors to evaluate the plant products of agricultural biotechnology. The first session described risk assessment and analysis, its reliance on credible data and information and how biosafety research is funded by governments. The speakers in this session provided insight into the development of regulatory systems for transgenic plants based upon science-based risk assessment; how the critical issue of uncertainty can be dealt with in risk assessments; the 
availability of international guidance for risk assessment; and how and why biosafety research on transgenic organisms in the environment is funded by governments. This paper is a summary of the presentations and discussion in Session I.

\section{RISK ASSESSMENT, UNCERTAINTY AND SCIENTIFIC DATA AND INFORMATION}

Risk assessors and decision makers have worked with the scientific community since the first transgenic organisms were proposed for field testing in the 1980s. The scientific community has provided leadership in framing the approach to risk assessment of transgenic organisms using the umbrella of the risk analysis paradigm that includes risk assessment and risk management and can include risk communication. The scientific community introduced the concept of familiarity, emphasizing the need for understanding of the unmodified organism, the trait and the organism that donated the genetic material, as well as the recipient environment into which the modified organism is to be used in order to identify potential hazards on a case-by-case basis (NAS, 1989; Tiedje et al., 1989). These concepts allowed a framework to evaluate transgenic organisms to be constructed and elaborated internationally.

As the application of biotechnology to agricultural products evolved, research to define and observe the likely effects of these products in the environment has been critical. For assessors, it has also emphasized the need for discipline in making the distinction between that evidence which is important for determining whether a hazard or a risk is present and that which is spurious or aberrant and does not contribute to the weight of evidence necessary to make an appropriate decision.

Sue Meek, the Australian Gene Technology Regulator, provided an overview of risk assessment within a national regulatory structure (Meek and Keese, 2006). The challenges faced by regulators are embedded in the scope and boundaries of the authorizing legislation in each country. The structure of the regulatory system varies from country to country due to specific economic, social and environmental factors. In addition, the quality and quantity of data that is necessary and sufficient to meet the needs of each country vary.

For the products of modern biotechnology, it has been difficult to respond to values in the process of setting up and implementing a regulatory system, including essential components such as an understanding of what is 'harm' and what is an acceptable risk. The regulatory system in each country has to achieve credibility and establish trust, and this is complex because of technical and arcane terminologies as well as varying stakeholder assumptions, beliefs, and agendas. To establish trust, the
Australian Office of the Gene Technology Regulator has spent many years developing a system of advisory committees to review technical aspects of the system and many processes for public consultation and consultation with many governmental agencies for both policies and decisions.

Globally, most risk assessments are qualitative. Qualitative assessments are just as credible as quantitative assessments and are advantageous currently, as long-term data sets are not (yet) available. Risk assessment methodologies have common approaches between countries even though the details of their implementation may vary. An example of such variation occurs with the baseline information that is used for comparison with the use of the transgenic plant, which may vary as well as the endpoints to be evaluated, particularly as some endpoints examined are hard to define and measure. In addition, there have not been agreed upon standards as to what constitutes "safety" or "safe" and it is problematic that different risk assessors use divergent information sources which may vary in quality.

Uncertainty and the concern for caution is a major challenge for regulators and is a key to stakeholder confidence. As it is impossible to have 'complete and perfect' knowledge, there is always an element of uncertainty in risk assessment. However, good data helps reduce uncertainty. Any data used as a basis for decisions is only relevant for regulatory systems if it is credible, reliable, and available. Data needs to be sufficient as well. Thus, discrimination by the risk assessor is needed in determining whether increasing amounts of data and information will provide a concomitant increase in the ability to address a particular issue. As a result, expertise and resources are needed to understand and effectively apply the available data in risk assessment.

According to Keith Hayes of Australia's CSIRO, the assumptions and kinds of uncertainty should be identified at the start of the assessment process (Hayes et al., 2006). The risk assessor should consciously understand how these assumptions and kinds of uncertainty are taken into account in the risk assessment. A credible risk assessment should be faithful to the assumptions and kinds of uncertainty embedded in it.

To deal with uncertainty effectively, it is helpful to examine what the sources of uncertainty are. These should be made explicit. This means defining uncertainty appropriately and describing or understanding what will reduce it. To effectively deal with uncertainty, the cost of reduction and whether it serves the purpose of the assessment must be accounted for. Uncertainty can be addressed in a number of ways, depending upon the circumstance - by using concise and clear language, doing more experiments, and being clear with how uncertainties are being dealt with. 
Linguistic uncertainty, uncertitude and variability are the three types of uncertainty associated with environmental risk assessment which must be addressed. Linguistic uncertainty is embedded in the use of vague language and is used more in qualitative assessments. For example, the use of the term 'large-scale' can mean different things depending on the context. Terminology such as 'high', 'medium', and 'low' builds in problems in evaluation and uncertainty at the boundaries between the different categories. An insignificant difference can mean change from one category to another. However, with genetically engineered organisms, there has been no demonstration of a 'small' change resulting in a 'large' consequence. In addition, there are not crisp, clear definitions of categories and it is difficult to quantify/understand the differences between such categories. If no information is available, then assessors can and often do move to best or worst case boundaries (or scenarios) to accomplish the assessment. Linguistic uncertainty is not eliminated with quantitative assessments.

Variability can't be reduced, just understood better. Models can be developed to address variability and uncertitude but models are functions of variable parameters and the parameters themselves might be uncertain with their own distributions. The understanding, or lack thereof, of the dependency or independency of variables also can have an impact on the usefulness of a model. If there is not enough information to understand the meaning of the variability, then one can use the best and/or worst case bounds but this too, is not without problems.

Different types of models each have advantages and limitations: statistical models are precise, general and not realistic; process models are precise, not general, but realistic; and qualitative models are general, realistic, but not precise. Precision does not necessarily mean accuracy while realism implies both accuracy and precision. Models are hard to translate as they are usually case-specific. There are not many quantitative studies dealing with uncertainty for transgenic organisms, perhaps because of the tendency to do qualitative assessments. According to Hayes, model uncertainty is the " 800 pound gorilla in the middle of the room".

Ryan Hill from the Secretariat of the Convention on Biological Diversity, elaborated the international approaches to risk assessment of transgenic plants (Hill, 2006). The fundamental definitions for environmental risk assessment of transgenic plants are generally agreed upon internationally. The terminology for these varies, but the concepts and fundamental elements are the same in the various venues that have worked in this arena over the years: the United Nations Environment Program, the International Plant Protection Convention, the Organization for Economic Cooperation and Development, and the Cartagena Protocol for Biosafety. All of the accepted guidance use the same fundamental principles to address what constitutes a risk assessment and what it should do.

The entire process of risk assessment, management and communication is iterative in the development of a decision about a product or class of products as well as in the evolution of policies by governments and international organizations. The iterative nature of risk assessment can begin with an initial 'quick-and-dirty' worst case assessment followed by a tiering of detail (data and information) needed based upon the initial assessment. The level of detail needed will be proportional to the potential risk presented. Once risk is initially assessed, it can be re-evaluated based upon the mitigation measures in place or applied. In other words, the presence of risk mitigation or management measures such as those that may already be a part of the agricultural system, are taken into account during the risk assessment. It should be recognized that baselines for comparison change over time as, for example, agricultural practices change and thus the entire process for assessing a class of products can evolve. Most assessment approaches rely upon familiarity with the organism while evaluating a changing receiving environment but may not explicitly state this.

Some assessment frameworks recommend that more data be obtained to address uncertainty - the Sanitary and Phytosanitary Agreement and the Cartagena Protocol on Biosafety. Without addressing uncertainty, there is a poor characterization of risk. It must be recognized, however, that a reduction in uncertainty does not necessarily change the subsequent decision that is made. Two decision-makers can reach opposite conclusions based upon the same risk assessment. Risk assessment and precaution are not incompatible; risk assessment is not the opposite of precaution. Risk assessments are functional and are part of the decision-making process but not its entirety. Precaution can come in at the decision-making level after the risk assessment is done. The level of risk aversion used by the decision-maker is based upon the values in the context in which the decision is being made.

Most assessment frameworks rely upon the use of multiple sources of scientific information or a weight of evidence approach for most of the steps in risk assessment - hazard identification, mitigation measures, for example. Steps by scientists to assure that biosafety research is used by policy makers globally can include engagement internationally through participating in the development of guidance documents, aiding developing countries with risk assessments (as representatives of governments and organizations), and through presentation of side-events at the Meeting of the Parties of the Cartagena Protocol on Biosafety.

The Cartagena Protocol on Biosafety has a Roster of Experts that was established to aid developing countries in doing risk assessments and scientists need to be put 
forward by their governments to participate in the area of risk assessment. The Roster of Experts needs to be populated by experts of credible standing in the research and assessment worlds in order to have a productive impact on developing countries that are struggling to set up credible risk assessment processes. Researchers can get on the Roster of Experts by working with their national governments to be put forward as a nominee.

\section{RISK ASSESSMENT AND FUNDING OF BIOSAFETY RESEARCH: RESEARCH PRIORITIES}

Research to support risk assessment is a significant and needed investment for governments. How the priorities for this investment are determined can involve complex processes that include the risk assessors responsible for review of transgenic organisms as well as policy and decision-makers. After the initial talks on risk assessment and the use of scientific data and information, Session I described how research priority setting has been approached in the United States (U.S.), the European Community, and for research funding dedicated to developing countries.

The U.S. Agriculture Risk Analysis Task Force (AGRA), headed by Rita Teutonico of the U.S. National Science Foundation, was set up in 2003 to link the U.S. regulatory and the U.S. public research agencies to develop research priorities for risk analysis of transgenic organisms (Teutonico, 2006). Research priorities were identified that focus on general principles, or classes of products either in current or imminent commercialization, rather than for specific products. Much data and information on specific products is already developed by private industry. In addition, AGRA identified that it is critical for the U.S. government to address evolving or emerging regulatory issues; new types of products bring new issues.

As part of AGRA, the regulatory agencies first identified their needs, which were then used to survey the many U.S. research agencies in order to understand the kinds of research being done by the U.S. research agencies in the areas identified. Almost twenty agencies were involved. For plant risk assessment research, AGRA identified the following informational needs: toxicology and allergenicity studies of novel proteins; unintended biological effects of plant-incorporated protectants, invasiveness and weediness; molecular impact of transgenes; stable expression and persistence of transgenes; genetic outcrossing in plants; and outcrossing reduction in plants. Once the needs were identified and an understanding of what kinds of research was being done to meet those needs was reached, AGRA then identified opportunities for further investment in areas such as weediness and invasiveness as well as allergenicity and toxicity. Research needs for animal risk assessment research and risk management of agricultural products were also identified. After these assessments were complete, AGRA hosted a conference with regulators and researchers that were federally funded with presentations by the latter. Research topics presented were within the categories identified by AGRA.

In the future, AGRA thought it would be useful to take this process further and incorporate the opinions about research priorities of the scientists that have worked in these areas. In addition, to develop a comprehensive understanding of the information available and thus what is still needed, an overview is needed to take into account the data generated by industry that addresses topics of importance.

Several agencies within the U.S. government have ongoing dedicated research and grant programs to address the needs of regulators of transgenic products. These programs include processes to identify and prioritize the research needs of regulators. The agencies include the U.S. Environmental Protection Agency's Office of Research and Development, the U.S. Agency for International Development, and the U.S. Department of Agriculture's Agriculture Research Service, and Cooperative State Research, Education and Extension Service. The AGRA process facilitated the coordination and interaction between these and other research agencies and the regulators.

The European Community has been funding research on the biosafety of genetically modified organisms for 21 years. According to Ioannis Economidis of the European Commission (EC), the strategy of funding has shifted over time to fewer but larger projects developed and implemented by consortia of researchers and institutions (Economidis, 2006). This research is complementary to testing and regulatory requirements within the Community itself. Relevance to risk assessment is an informal part of biotechnology research. Regulation, research itself and management are all impacted by the results.

A major question that arose in discussions of funding within the EC was "what issues have been closed through this research?' As a result, funding is moving away from biosafety research because of the inability of the research to resolve issues - the endpoints of the research are not clear. In addition, it is not clear how the results of all of this research have helped in decision making. Monies are moving towards areas such as the 'Knowledge-based Bioeconomy' and areas that are perceived to enhance European competitiveness.

The general conclusions of the European Community about biosafety of transgenic products are fairly 
clear however. They are the following: genetic modification is not inherently safe or unsafe; no new risks have been presented; food safety has been clarified; questions about conventional agriculture have been raised as the result of concerns over transgenic products; concerns remain about the environment; biotechnology has allowed elaboration of new knowledge of molecular biology, environmental impacts and ecology; there is a need to be predictive; and communication about most aspects of the technology and products is still a great challenge.

Hector Quemada described the U.S. Agency for International Development's Biotechnology and Biodiversity Interface Program that provides major funding for biosafety research in developing countries (Quemada and Hokanson, 2006). The purposes of this research are to support the structure, function and goals of national regulatory systems for risk assessment. The funding helps develop the goals of research, the knowledge base to establish a regulatory agency, and the ability to do risk assessment, as well as post-commercialization monitoring. Animals and vaccine development are not funded. The projects to date have addressed gene flow of crops in Africa, outcrossing and non-target impacts in Brazil and the Philippines.

An important question for developing countries that have limited resources to dedicate to risk assessment and regulatory systems is whether there is any evidence of any harm from transgenic plants anywhere. This question is particularly important for developing countries, as there is a difference, depending upon the country, as to who pays for risk assessment and research. In the industrialized countries, companies generate data for particular products, and the public pays for more generalized research through government granting programs. In developing countries, products are paid for by the country itself. Products are not developed for profit, so regulatory packages are developed and paid for by the state or research agencies, and thus it is critical for them to focus the use of resources.

\section{CONCLUSIONS}

The initial session of the symposium set the stage for discussion at the symposium of the interaction of biosafety research and risk assessment, through introducing national and international guidance on risk assessment, to show how critical direction is provided about the types of scientific data needed to evaluate transgenic organisms. A major issue for risk assessors is the identification and addressing of uncertainty, and there are different types of uncertainty associated with relevant data and information, depending upon how it is used and how the risk assessment is to be performed. Guidance for risk assessment as well as risk assessment itself is strongly influ- enced by the results of biosafety research. And, the type of research that is funded by governments is many times identified by risk assessors or in collaboration with risk assessors. The processes by which nations and regions identify biosafety research priority areas were described.

In preparation for the 9th ISBGMO, the International Society for Biosafety Research did a survey of its members in 2006 to get a better understanding of what kind of biosafety research was deemed important. There was a strong undercurrent in responses indicating that it has to be very clear what information is 'need to know' versus 'nice to know' in order for risk assessment to be most effective and efficient.

Scientific research is the foundation for expanding the knowledge base used in risk assessments and, as such, is essential for performing robust and credible assessments necessary for making sound decisions. Biosafety research is largely funded and performed to provide concepts, models, and data that allow biosafety issues to be defined and analyzed and uncertainty to be understood. Understanding the potential for adverse environmental effects from transgenic organisms and the characterization of associated risks depends not only on quality of biosafety research but also on ongoing interaction between risk assessors, regulators and researchers.

\section{DISCLAIMER}

Sally L. McCammon, Ph.D., is the Science Advisor at BRS, APHIS, USDA. The views expressed in this paper are those of the author and do not necessarily represent those of the United States Government.

\section{REFERENCES}

Economidis I (2006) Two decades of EC sponsored research on safety of GMOs. In Roberts A, ed, Proceedings of the 9th International Symposium on the Biosafety of Genetically Modified Organisms. http:// www.isbr.info, pp 42-44

Hayes K, Regan H, Burgman M, Devlin R, Kapuscinski A, Dambacher J (2006) Methods to address uncertainty in ecological risk assessment of genetically modified organisms. In Roberts A, ed, Proceedings of the 9th International Symposium on the Biosafety of Genetically Modified Organisms. http://www.isbr.info, pp 28-31

Hill R (2006) Principles for risk assessment - an international perspective. In Roberts A, ed, Proceedings of the 9th International Symposium on the Biosafety of Genetically Modified Organisms. http:// www.isbr.info, pp 32-37

Meek S, Keese P (2006) Putting theory into practice - applying risk analysis to the regulation of GMOs. In Roberts $\mathrm{A}$, ed, Proceedings of the 9th International 
Symposium on the Biosafety of Genetically Modified Organisms. http://www.isbr.info, pp 23-25

National Academy of Sciences (1989) Field Testing Genetically Modified Organisms: Framework for Decisions, $170 \mathrm{p}$

Quemada H, Hokanson K (2006) Biotechnology and Biodiversity Interface competitive grant program. In Roberts A, ed, Proceedings of the 9th International Symposium on the Biosafety of Genetically Modified Organisms. http://www.isbr.info, p 45
Tiedje J, Colwell R, Grossman Y, Hodson R, Lenski R, Mack N, Regal P (1989) The planned introduction of genetically engineered organisms: ecological considerations and recommendations. Ecology 70: 298-315.

Teutonico R (2006) Setting priorities for risk assessment research. In Roberts A, ed, Proceedings of the 9th International Symposium on the Biosafety of Genetically Modified Organisms. http:// www.isbr.info, pp 38-41 